\title{
EXPERIMENTAL STUDIES ON THE VALUE OF ARCHERY IN PARAPLEGIA
}

\author{
By Sir Ludwig Guttmann and Dr N. C. Mehra \\ National Spinal Injuries Centre, Stoke Mandeville Hospital, Aylesbury
}

DURING World War II one of us (L. G.) introduced sport as part of the medical treatment of paraplegics and tetraplegics; archery was one of the first events in clinical sport. As a remedial exercise it proved ideal for training those muscle groups above the level of the spinal cord lesion, in particular, the arm, shoulder and trunk muscles-the muscles mainly used in archery. The pullweight of the bow varies between 36 and $42 \mathrm{lbs}$. for men and 25-38 lbs. for women, children, naturally, use lighter pullweights. The training of the trunk muscles, in particular trapezius with its adjustment to the I2th dorsal vertebra, enables the paraplegic to restore and maintain his upright position without the aid of artificial corsets made from steel and leather, thus developing a physiological corset. In due course, these muscles develop a hypertrophy as shown in Figure 5, B. Archery has great fascination, as the archer accomplishes everything by his own judgement and strength, nothing is done for him. In contest with himself, he improves his performance and overcomes fatigue, that important symptom in the early stages of physical readjustment and rehabilitation. Moreover, archery is one of the sports events in which the paraplegic can compete on equal terms with the able-bodied, even in competition for internationally recognised long distance rounds. In fact, there are paraplegic men and women who have won the F.I.T.A. star for gaining over I000 points in the internationally recognised longest round: 36 arrows each at distances of $90,70,50$ and 30 metres (women 70,60, 50 and 30 metres). If one multiplies the pullweight of the bow by the number of arrows shot in this competition, one gets an idea to what tremendous physical efforts these wheelchair athletes are capable.

Even tetraplegics with paralysis of all fingers but with good function of the extensors of the wrist can take part and enjoy this sport. Those who have functioning triceps are provided with a glove to hold the hand onto the bow; those without functioning triceps are supplied in the addition, with an extension splint on the forearm. The drawing hand is provided with a slightly curved hook of light metal on the palmar surface of the glove fitted to the palm of the hand. With the aid of these appliances, the bow string can be drawn back and then, by twisting the hand, the hook is released from the bow string, thus releasing the arrow (figs. I-3). Although tetraplegics start, like paraplegic beginners, with the easiest round, called the St. Nicholas round ( 48 arrows each at 40 and 30 metres), by intensive training they succeed in shooting longer distances and some of them even the F.I.T.A. round.

It seemed to be worthwhile to study electromyographically the alternative muscle groups mainly involved in archery. Nine male paralysed subjects with complete spinal cord lesions at levels ranging from C.6 to T.I2 were studied. Surface electrodes were applied to the following muscles using a four-channel electromyograph furnished with photographic equipment: Trapezius (TRAP), 

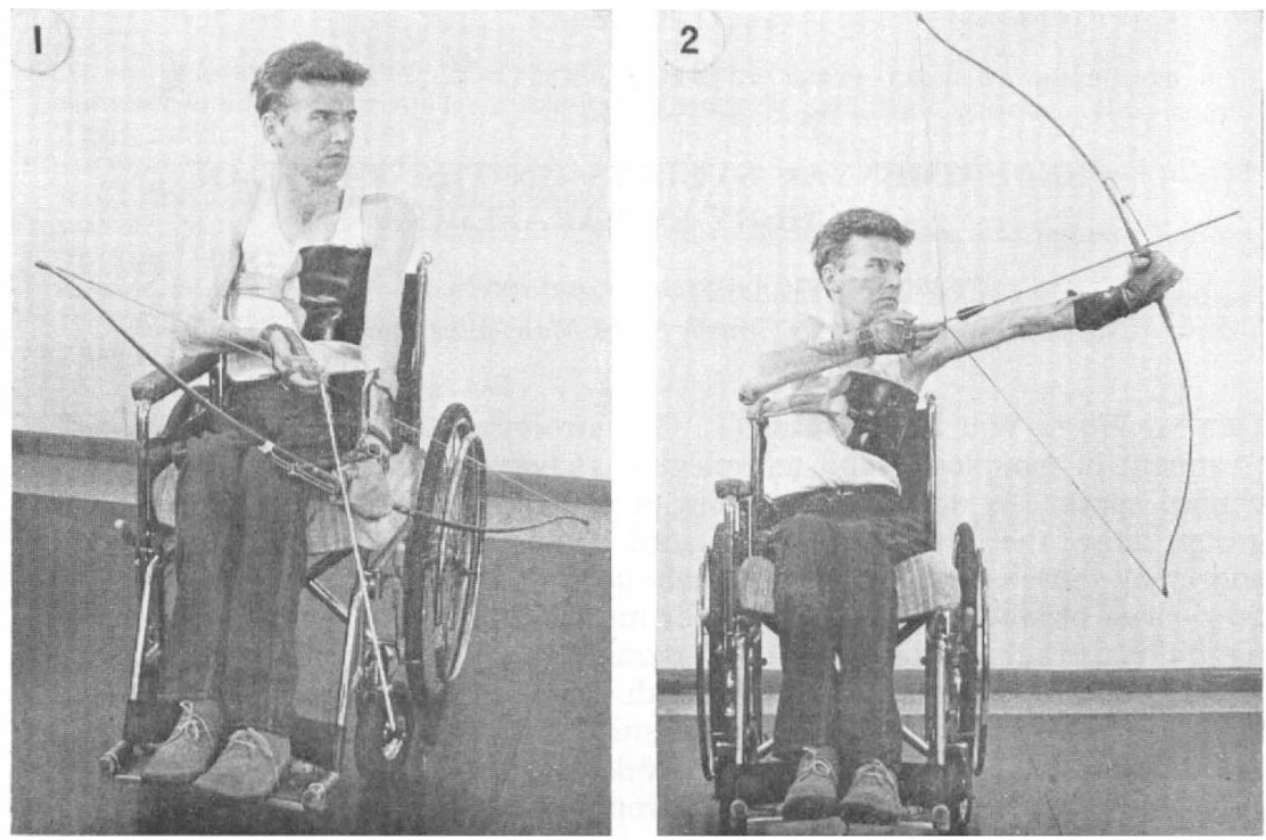

Fig. I.-Demonstrates the hook attachment on the palm of the hand to the bow in a tetraplegic patient below C. 6 with paralysis of all finger muscles but preserved extension of the wrist.

Fig. 2.-The same patient pulling the bow and with trunk supported to preserve balance.

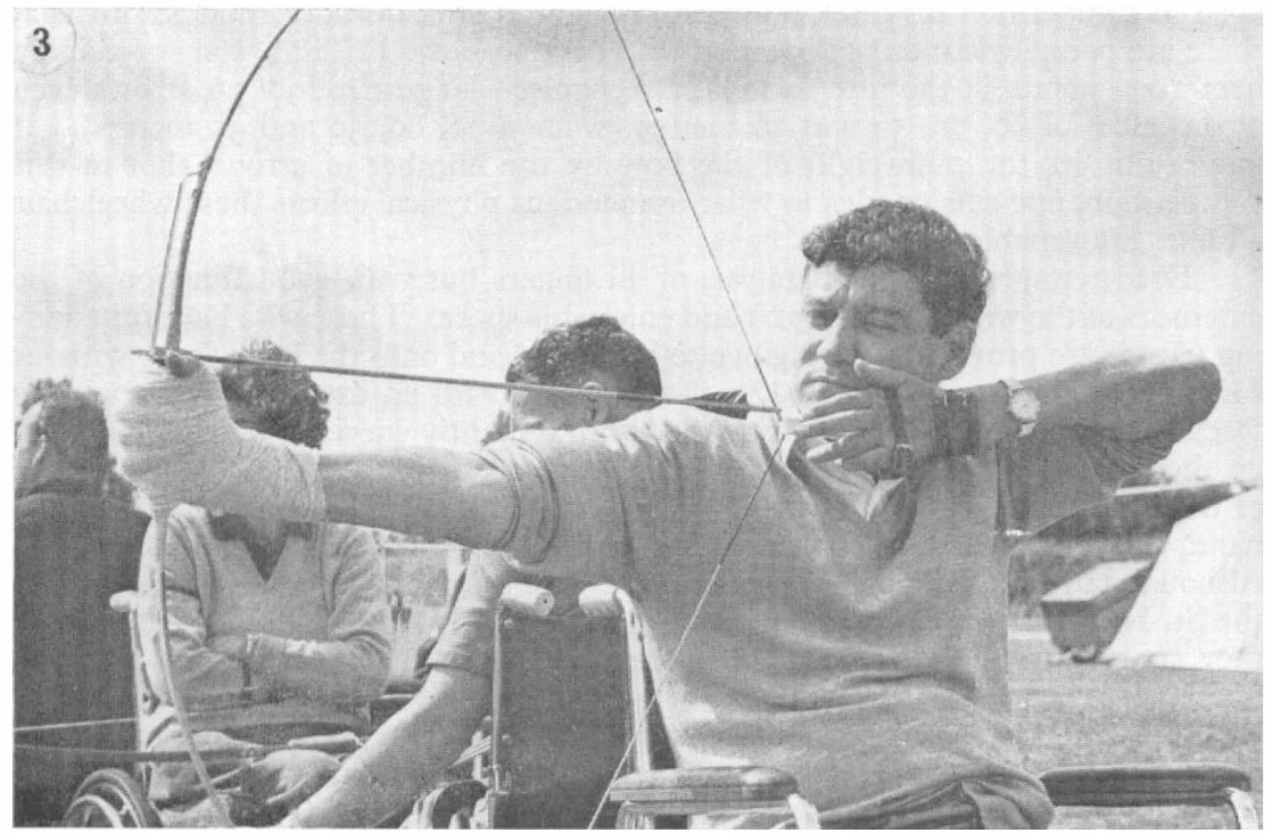

Fig. 3.-Another tetraplegic patient with good triceps but paralysis of the fingers, the hand fixed by a bandage on the bow. This picture shows the end of the hook attached to the bowstring before release by extension of the wrist. 


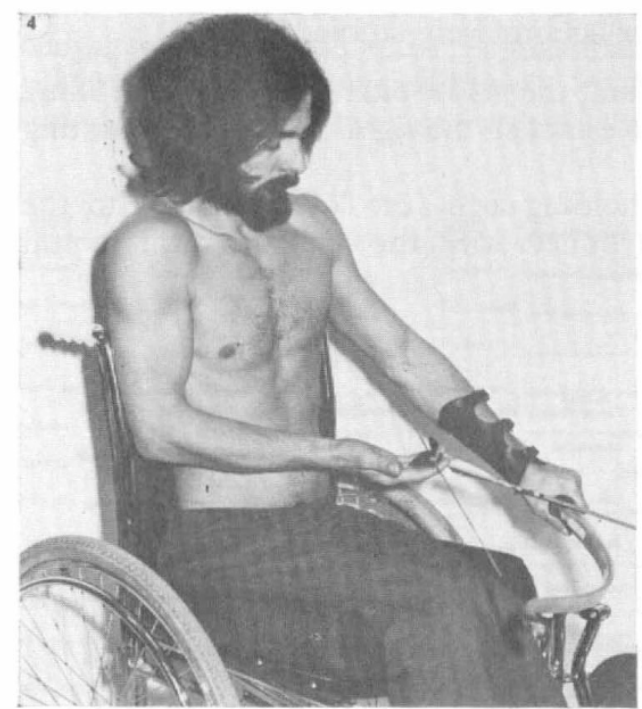

FIG. 4

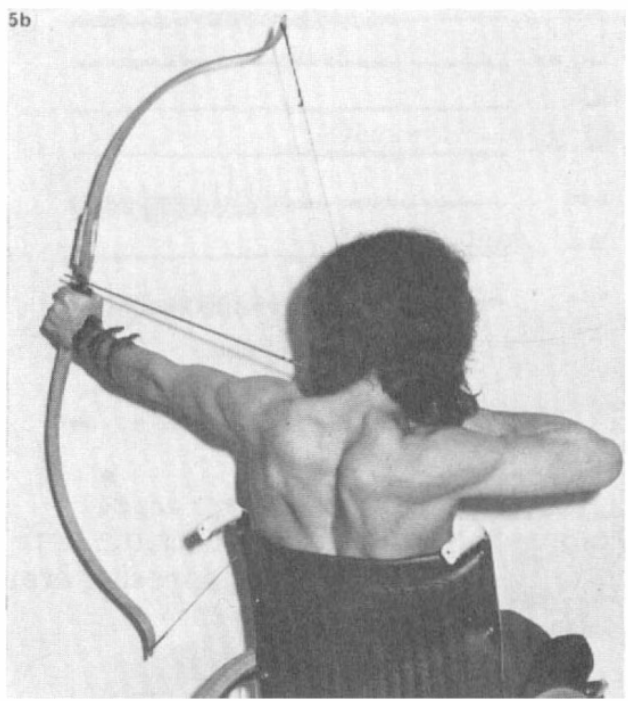

FIG. 5, B

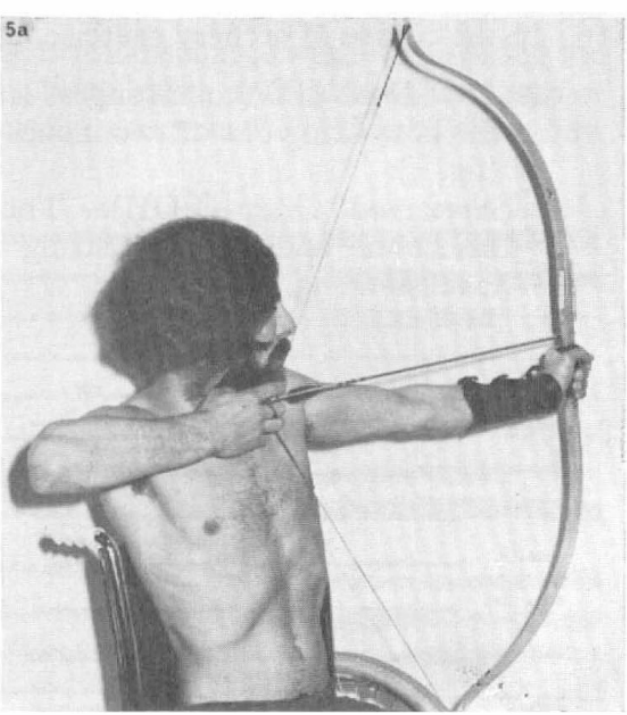

FIG. 5, A

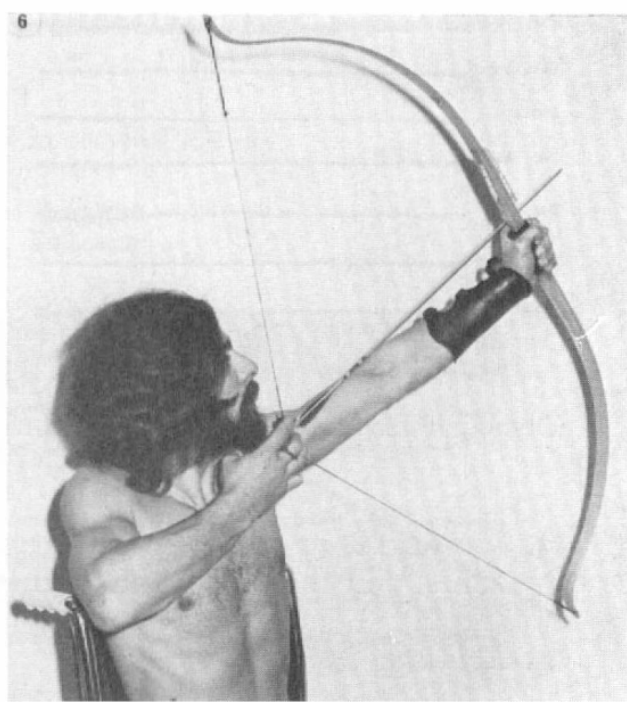

FIG. 6

Fig. 4.-Demonstrates the position of loading the arrow onto the bow and pulling the bow slightly to establish the proper position for shooting.

Fig. 5.-A, Shows the position in pulling the bow in horizontal draw. B, Shows the profound muscle action and hypertrophy of the shoulder muscles.

Fig. 6.-Demonstrates the vertical draw. 
Rhomboids (RHOM), Latissimus dorsi (LAT DO), Pectoralis major (PECT), Serratus anterior (SERR), Deltoid (DLT), Biceps (BIC) and Triceps (TRIC).

In the course of archery exercise, four actions were studied:

I. First Draw (ID) consisting of loading the arrow onto the bow and pulling the bow string one or two inches to establish the right position for shooting (fig. 4).

2. Horizontal Draw (FDH). The holding arm (the left) is raised to the horizontal and the bow string is pulled with the opposing arm to full extension (fig. 5, A and B).
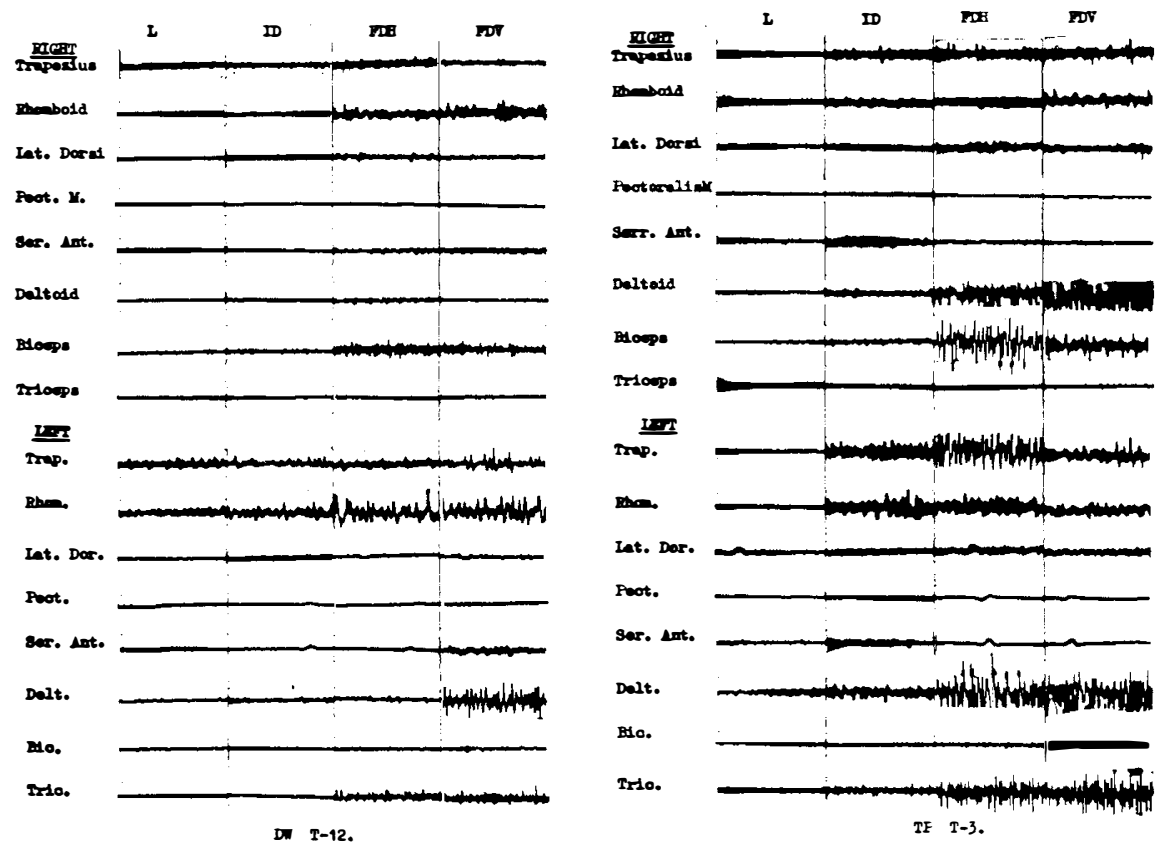

FIG. 7

Demonstrates the muscle action potentials (MAP) in a paraplegic below T.I2 as compared with a paraplegic below T.5.

3. Vertical Draw (FDV). The arm holding the bow is raised over the horizontal and the bow string is drawn to full extent with the opposing arm (fig. 6).

4. Releasing the Arrow.

Tetraplegics have to be strapped to the chair to maintain their equilibrium. Two of the subjects studied had an extension splint applied to the left elbow because of paralysis of the triceps.

Figures 7 and 8 demonstrate the electrical activity of the individual muscles in the thoracic lesions as compared with the cervical lesions involved in the archery exercise. There was, as one would expect, great variability in the intensity of action potentials and every one of the subjects exhibited different degrees of 
electrical discharge whatever the level of the lesion. However, there was some uniformity in other respect: (a) The lowest electrical discharge occurred during loading and first draw, while the maximal electrical discharge was found during the full horizontal and vertical draws. (b) A sudden sharp and maximal increase of wave form as a rebound occurred in all muscles concerned on release of the bow
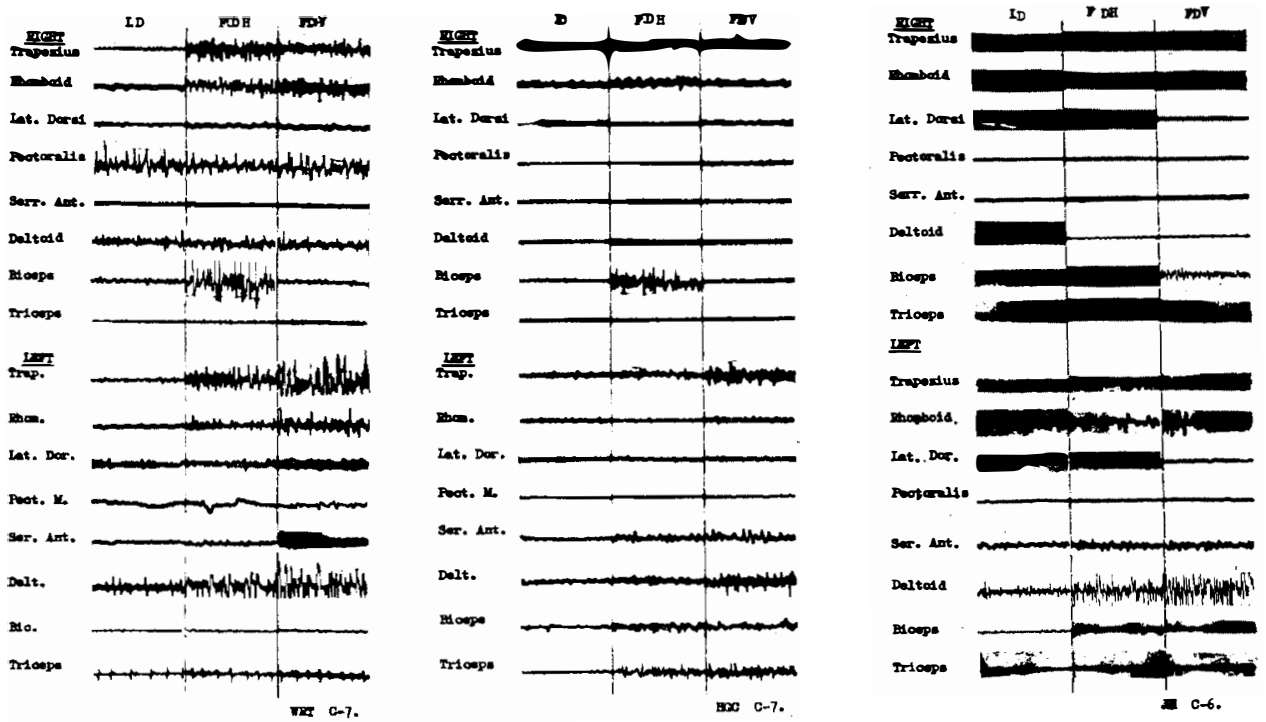

Fig. 8

Demonstrates the MAP in cervical lesions.

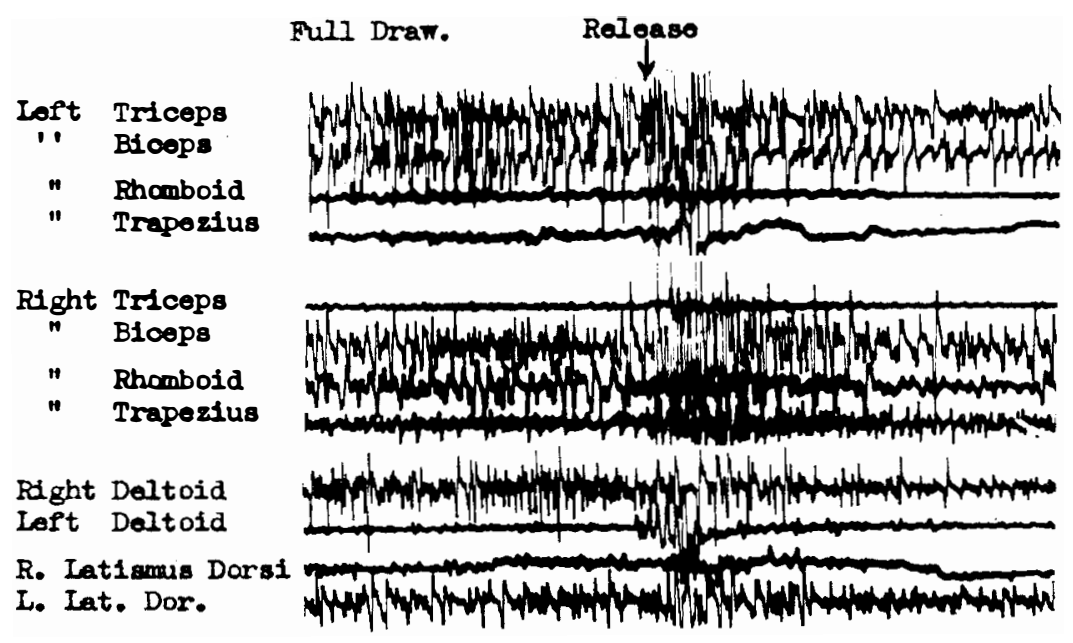

Effeots of release of bowstring on waveforms.

Fig. 9

Shows the effect of releasing the bowstring on action potentials. 
string before the electrical discharge slowed and ceased (fig. 9). (c) Another uniformity was found in the low level or even absence of electrical discharge of the latissimus dorsi, serratus anterior and pectoralis major with the exception in one subject with a C.7 lesion where the right pectoralis major exhibited a high level of electrical activity.

In evaluating the results further, it appeared that certain muscles showed preponderence in their activity during archery. This applies in particular to the

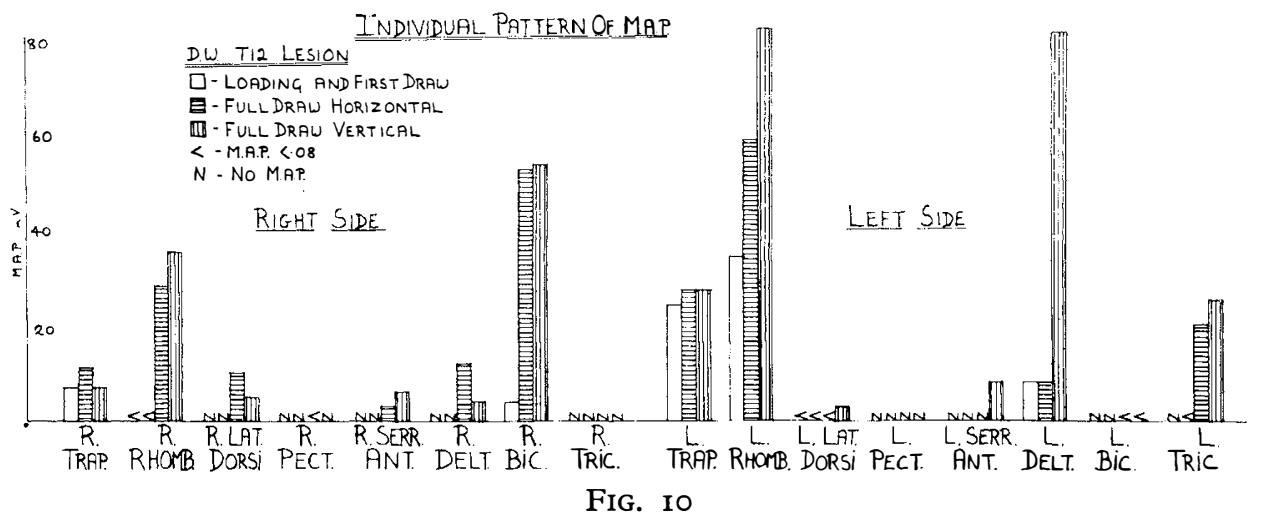

Demonstrates the individual pattern of MAP in a T.I2 lesion.
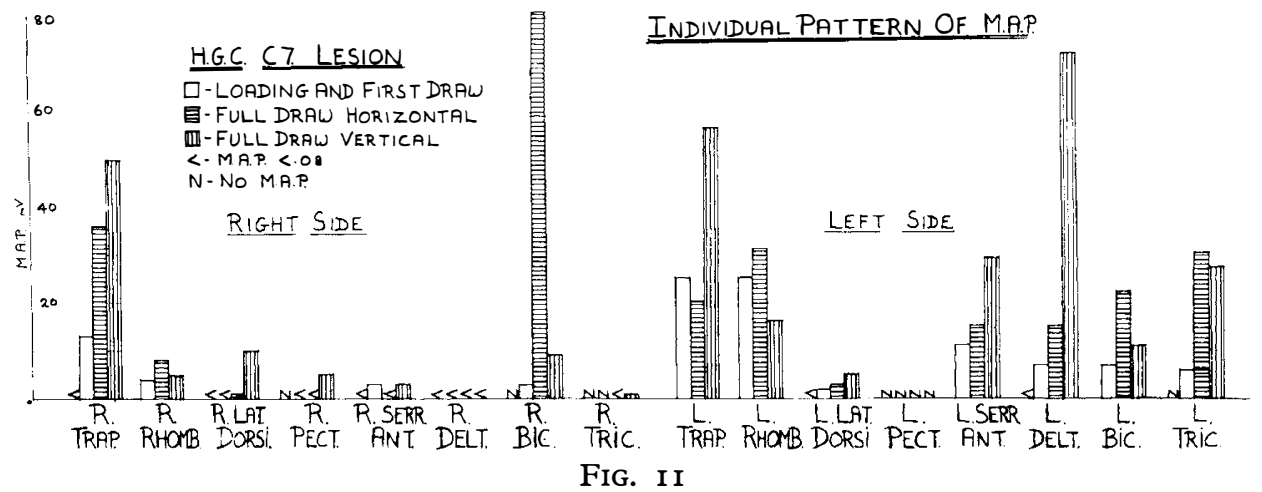

Shows the individual pattern of MAP in a C.7 lesion.

deltoid on both sides in securing in horizontal and vertical positions of the arms. That the biceps on the drawing right arm (in the right-handed subjects) and the left triceps on holding the bow in extension, show high electrical discharge is obvious. However, in the two cervicals supplied with extension splints because of the paralysis of the triceps, the electrical discharge was minimal, or absent. In all cervical lesions and also in the T.3 lesion trapezius and rhomboids, showed particularly marked electrical activity as compared with the T.I2 lesion. This indicating the importance of these muscle groups in bracing the shoulders in these high lesions (figs. IO-I2). 


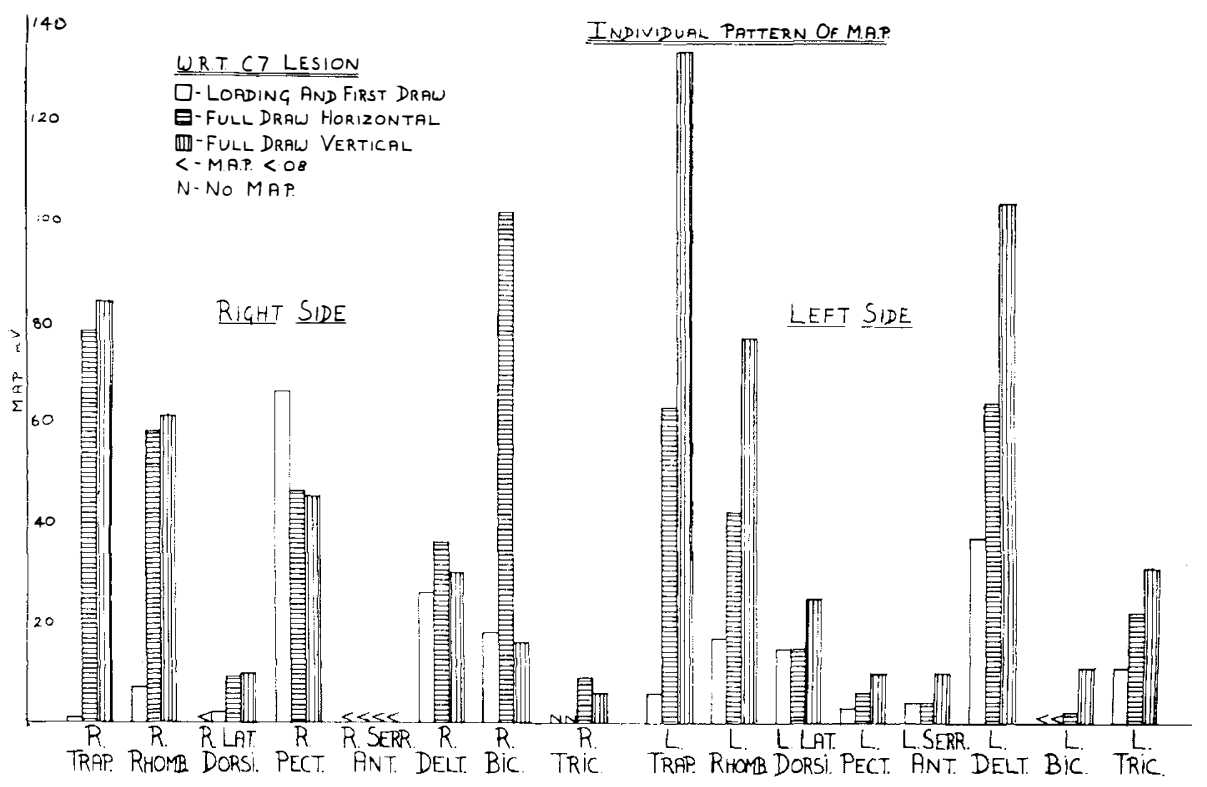

FIG. I2

Shows the individual pattern of MAP in another C. 7 lesion.

In conclusion, this study has been useful in clarifying the activity of muscle groups mainly involved in the various phases of archery sport. It was of particular interest to find that of the trunk muscles, latissimus dorsi and serratus anterior, in contrast to trapezius and rhomboids, play little part, if any, in this type of sport. 